\title{
Detection of 2-Hydroxyglutarate by 3.0-Tesla Magnetic Resonance Spectroscopy in Gliomas with Rare IDH Mutations: Making Sense of "False-Positive" Cases
}

\author{
Manabu Natsumeda ${ }^{1, *}\left(\mathbb{D}\right.$, Hironaka Igarashi ${ }^{2, *} \mathbb{0}$, Ramil Gabdulkhaev $^{3} \mathbb{D}$, Haruhiko Takahashi ${ }^{1}$, \\ Kunio Motohashi $\left.{ }^{1}{ }^{(}\right)$, Ryosuke Ogura ${ }^{1}{ }^{(0}$, Jun Watanabe ${ }^{1}\left(\mathbb{D}\right.$, Yoshihiro Tsukamoto $\left.{ }^{1}{ }^{(}\right)$, Kouichirou Okamoto ${ }^{4}$, \\ Akiyoshi Kakita ${ }^{3}{ }^{(}$, Tsutomu Nakada $^{2}$ and Yukihiko Fujii ${ }^{1}$
}

check for

updates

Citation: Natsumeda, M.;

Igarashi, H.; Gabdulkhaev, R.; Takahashi, H.; Motohashi, K.;

Ogura, R.; Watanabe, J.;

Tsukamoto, Y.; Okamoto, K.;

Kakita, A.; et al. Detection of

2-Hydroxyglutarate by 3.0-Tesla

Magnetic Resonance Spectroscopy in

Gliomas with Rare IDH Mutations:

Making Sense of "False-Positive"

Cases. Diagnostics 2021, 11, 2129.

https://doi.org/10.3390/

diagnostics11112129

Academic Editor: Silvia Morbelli

Received: 26 October 2021

Accepted: 15 November 2021

Published: 16 November 2021

Publisher's Note: MDPI stays neutral with regard to jurisdictional claims in published maps and institutional affiliations.

Copyright: (c) 2021 by the authors. Licensee MDPI, Basel, Switzerland. This article is an open access article distributed under the terms and conditions of the Creative Commons Attribution (CC BY) license (https:// creativecommons.org/licenses/by/ $4.0 /)$.
1 Department of Neurosurgery, Brain Research Institute, Niigata University, Niigata 951-8122, Japan; haruhiko.takahashi@icloud.com (H.T.); kmoto@bri.niigata-u.ac.jp (K.M.); oguryou@bri.niigata-u.ac.jp (R.O.); watanabejun1003@yahoo.co.jp (J.W.); yoshi.tsukamoto@me.com (Y.T.); yfujii@bri.niigata-u.ac.jp (Y.F.)

2 Center for Integrated Brain Sciences, Brain Research Institute, Niigata University, Niigata 951-8585, Japan; tnakada@bri.niigata-u.ac.jp

3 Department of Pathology, Brain Research Institute, Niigata University, Niigata 951-8585, Japan; ram.gab258852@gmail.com (R.G.); kakita@bri.niigata-u.ac.jp (A.K.)

4 Department of Translational Research, Brain Research Institute, Niigata University, Niigata 951-8585, Japan; oko-okamoto@bri.niigata-u.ac.jp

* Correspondence: natsumeda@bri.niigata-u.ac.jp (M.N.); higara@bri.niigata-u.ac.jp (H.I.)

\begin{abstract}
We have previously published a study on the reliable detection of 2-hydroxyglutarate (2HG) in lower-grade gliomas by magnetic resonance spectroscopy (MRS). In this short article, we re-evaluated five glioma cases originally assessed as isocitrate dehydrogenase (IDH) wildtype, which showed a high accumulation of $2 \mathrm{HG}$, and were thought to be false-positives. A new primer was used for the detection of $I D H 2$ mutation by Sanger sequencing. Adequate tissue for DNA analysis was available in 4 out of 5 cases. We found rare $I D H 2$ mutations in two cases, with IDH2 R172W mutation in one case and IDH2 R172K mutation in another case. Both cases had very small mutant peaks, suggesting that the tumor volume was low in the tumor samples. Thus, the specificity of MRS for detecting IDH1/2 mutations was higher (81.3\%) than that originally reported $(72.2 \%)$. The detection of 2HG by MRS can aid in the diagnosis of rare, non-IDH1-R132H IDH1 and IDH2 mutations in gliomas.
\end{abstract}

Keywords: 2-hydroxyglutarate; glioma; magnetic resonance spectroscopy; rare IDH mutations; false-positive

\section{Introduction}

Isocitrate dehydrogenase (IDH)-mutant gliomas produce the oncometabolite 2-hydroxyglutarate ( $2 \mathrm{HG}$ ). We previously reported on the reliable detection of $2 \mathrm{HG}$ by 3.0-tesla magnetic resonance spectroscopy (MRS) in a cohort of 52 lower-grade glioma patients (WHO grades 2 and 3) [1]. A cutoff of $1.489 \mathrm{mM}$ for $2 \mathrm{HG}$ yielded $100 \%$ sensitivity and a $72.2 \%$ specificity for the detection of IDH1 or IDH2 mutations was reported. A high level of $2 \mathrm{HG}$ was detected in 5 of 27 (18.5\%) gliomas that were determined to be IDH-wildtype. These were thought to be false-positive results or a failure to detect rare IDH1 or IDH2 mutations by DNA sequencing [1]. The unambiguous detection of 2HG (chemical shift $2.25 \mathrm{ppm}$ ) by MRS is difficult because of a spectral overlap with glutamate (Glu; $2.43 \mathrm{ppm}$ ), glutamine (Gln; 2.34 ppm), and gamma-aminobutyric acid (GABA; 2.28 ppm). Recently, the tumor recurred in one of the five patients, and the patient underwent a second surgery. An analysis of IDH1/2 mutations using Sanger sequencing revealed an IDH2 mutation. In the current study, we re-evaluated IDH1 and IDH2 status in the remaining "false-positive" 
cases with available tissue. We found that the $2 \mathrm{HG}$ detection by MRS was useful in the selection of glioma cases with rare IDH1 and IDH2 mutations.

\section{Materials and Methods}

MRI/1H-MRS was performed using a 3.0-tesla system (Signa LX, General Electric, Waukesha, WI, USA) as previously reported [1], in accordance with the human research guidelines of the Internal Review Board of Niigata University (Approval \#2017-0163) after obtaining written consent from all participants. Proton density images (Fast Spin Echo; $\mathrm{TR} / \mathrm{TE}=5000 / 40$; FOV: $20 \times 20 \mathrm{~mm}$; matrix: $256 \times 256$; slice thickness: $5 \mathrm{~mm}$; interslice gap: $2.5 \mathrm{~mm}$ ) were captured. The slice with the largest depiction of the tumor on proton density images was selected for SVMRS. A point-resolved spectroscopic sequence (PRESS) with a chemical-shift-selective water suppression was used with the following parameters: TR: $1.5 \mathrm{~s}$; TE: $30 \mathrm{~ms}$; data point 512; spectral width $1000 \mathrm{~Hz}$; number of acquisitions: 128-196; volume of interest (VOI): 12-20 × 12-20 × 12-20 mm). The volume of interest (VOI) was designed to minimize the suspected areas of necrosis and hemorrhage. A spectral analysis was performed using LCModel version 6.3 (Stephen Provencher, Oakville, ON, Canada) [2]. This software automatically adjusts the phase and chemical shift of the spectra, estimates the baseline, and performs eddy current corrections. Relative metabolite concentrations and their uncertainties were estimated by fitting the spectrum to a basis set of spectra acquired from individual metabolites in-solution. The basis set was provided by Dr. Steven Provencher [2] and was calibrated with an MRS phantom solution (18-cm-diameter MRS HDsphere, model 2152220; General Electric) using our MR system. Nineteen metabolites were included in the LCModel basis set, including alanine, aspartate, creatine (Cr), phosphocreatine (PCr), GABA, glucose, Gln, Glu, glycerophosphocholine (GPC), phosphocholine (PC), glutathione (GSH), 2HG, myo-inositol (Ins), lactate, $\mathrm{N}$-acetylaspartate (NAA), N-acetylaspartylglutamate (NAAG), scyllo-inositol, taurine, and guanine. Total NAA (tNAA: the sum of NAA and NAAG), total choline (tCho: the sum of GPC and PC), total creatine (tCr: the sum of $\mathrm{Cr}$ and PCr), and sum of Glu and Gln (Glx). To calculate the absolute metabolite concentrations, an unsuppressed water signal was used as a reference. Quantification estimates of $2 \mathrm{HG}$ were considered unreliable and were excluded when the Cramer-Rao lower bounds, returned as the percentage of standard deviation (\%SD) by LCModel, was greater than 35\%.

Sequencing for IDH1 and IDH2 was performed for four out of five cases with ample tissue for DNA analysis. DNA was extracted from fresh frozen tissue using the QIAamp Blood \& Tissue Kit (Qiagen, Valencia, CA, USA) or using a formalin-fixed, paraffin-embedded (FFPE) tumor tissue using the QIAmap DNA FFPE Tissue Kit (Qiagen). PCR amplification was performed using the following primer sets: forward $5^{\prime}$ ACCAAGGATGCTGCAGAAGC-3' ${ }^{\prime}$ and reverse 5'-AGATGGACGCCTATTTGTAAGT-3' at codon 132 for the IDH1 gene, and forward $5^{\prime}$-AGCCCATCATCTGCAAAAAC- $3^{\prime}$ and reverse $5^{\prime}$-CAGTGGATCCCCTCTCCAC-3' at codon 172 for the IDH2 gene [3], which were different from those used in the original study [1].

For fluorescence in situ hybridization (FISH) for $1 p / 19 q$ codeletion, formalin-fixed paraffin-embedded (FFPE) hematoxylin and eosin (HE)-stained tissue sections were examined to determine areas with high tumor cell density and a lack of necrosis and hemorrhage. Areas of interest on the HE-stained slides were transcribed to the reverse side of the unstained FFPE sections using a diamond-tipped marker. The FISH assay was performed on 4- $\mu$ m-thick sections, using ZytoLight SPEC 1p36/1q25 and ZytoLight SPEC 19q13/19p13 probes for locus-specific 1p and 19q analysis, respectively, following the manufacturers' instructions (ZytoVision, Bremerhaven, Germany). Additionally, 1p36 and 19q13 locusspecific red fluorescent probes were used as deletion targets and 1q25 and 19p13 green probes were used as internal controls. Nuclei were counterstained with 4,6-diamidino-2phenylindole (DAPI). FISH was performed on a Z-stacked two-dimensional image using a fluorescence microscope BZ-9000 (KEYENCE, Osaka, Japan). A minimum of 100 adjacent, non-overlapping interphase nuclei were examined for each assay. Whereas normal diploid 
nuclei displayed a signal ratio of $2 / 2$, a nucleus was considered to harbor a deletion if the target signal was 1 (i.e., 2/1) in relation to normal control signals. If the number of deleted nuclei was $\geq 50 \%$, the tumor was considered to present a deletion of the targeted chromosome part [4].

\section{Results}

\subsection{Rare IDH2 Mutation Detected in a Recurrent "False-Positive"Case}

In the original study, a 2HG of $1.489 \mathrm{mM}$ or higher was detected in 5 of $27(18.5 \%)$ gliomas that were determined to be IDH-wildtype. Recently, the tumor was found to have recurred in one of the five patients, and subsequently, the patient underwent a second surgery. A single voxel MRS, taken before the first surgery, showed characteristic small peaks at a chemical shift of approximately $2.25 \mathrm{ppm}$, reflecting 2HG (Figure 1A) and a high $2 \mathrm{HG}$ accumulation of $6.820 \mathrm{mM}$. A molecular analysis of the recurrent tumor via Sanger sequencing using a different $I D H 2$ primer revealed an $I D H 2$ R172W mutation (Figure 1B), and the pathological diagnosis was anaplastic oligodendroglioma, IDH-mutant and $1 \mathrm{p} / 19 \mathrm{q}$-codeleted. We re-analyzed the IDH2 status in the tumor from the initial surgery and found an IDH2 R172W mutation, albeit with a very small mutant peak (Figure 1C).

A

A

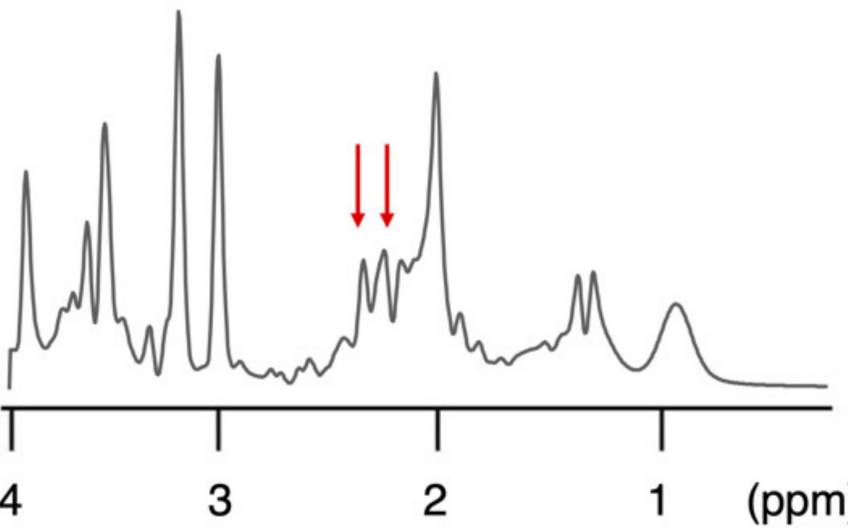

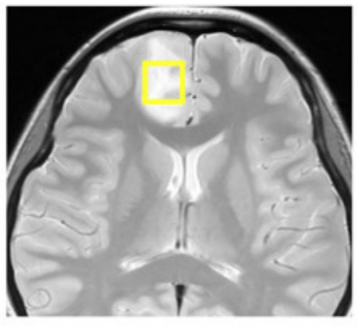

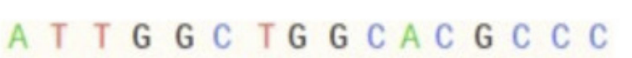

B

IDH2 R172W

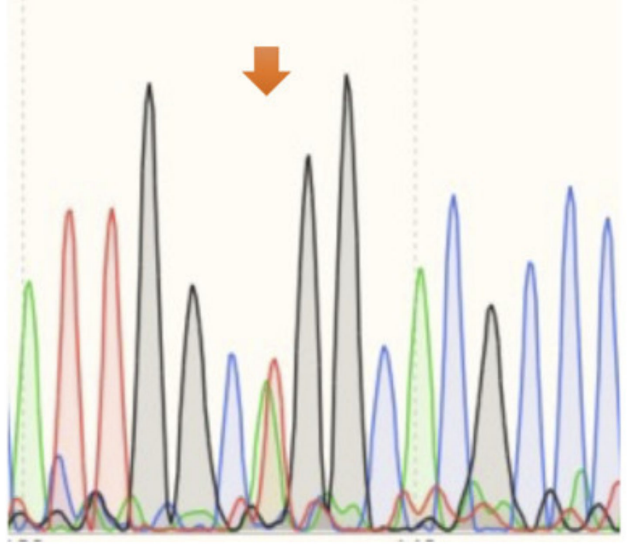

\section{IDH2 R172W}

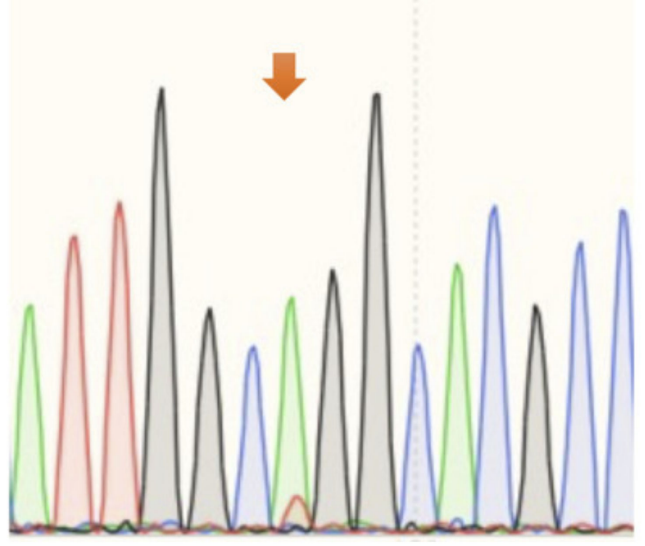

Figure 1. Rare IDH2 mutation diagnosed at relapse. (A) Single voxel MRS (SVMRS) spectra showing small peaks (red arrows) at a chemical shift of approximately $2.25 \mathrm{ppm}$ reflect the presence of 2-hydroxyglutarate (2HG). 2HG was quantified as $6.820 \mathrm{mM}$. (B) Obvious IDH2 R172W mutation was detected at relapse. (C) Reassessment of IDH2 mutation in the initial tumor revealed a small, mutant peak. 


\subsection{Re-Evaluation of IDH1 and IDH2 Mutations in Remaining Cases}

Subsequently, we re-evaluated the IDH1 and IDH2 status in three out of the four remaining "false-positive" tumors with available tissue and found a IDH2 R172K mutation in 1 patient with subtle peaks at a chemical shift of $2.25 \mathrm{ppm}$ (Figure 2A). A very small mutant peak was detected using Sanger sequencing (Figure 2B). Morphologically, perinuclear halos, chicken-wire vessels and calcification were observed, all of which are characteristic of oligodendrogliomas (Figure 2C). Furthermore, 1p (Figure 2D) and 19q (Figure 2E) deletions were confirmed by fluorescence in situ hybridization. The tumor was diagnosed as an oligodendroglioma, IDH-mutant and 1p/19q-codeleted, according to WHO2016 [5].

A
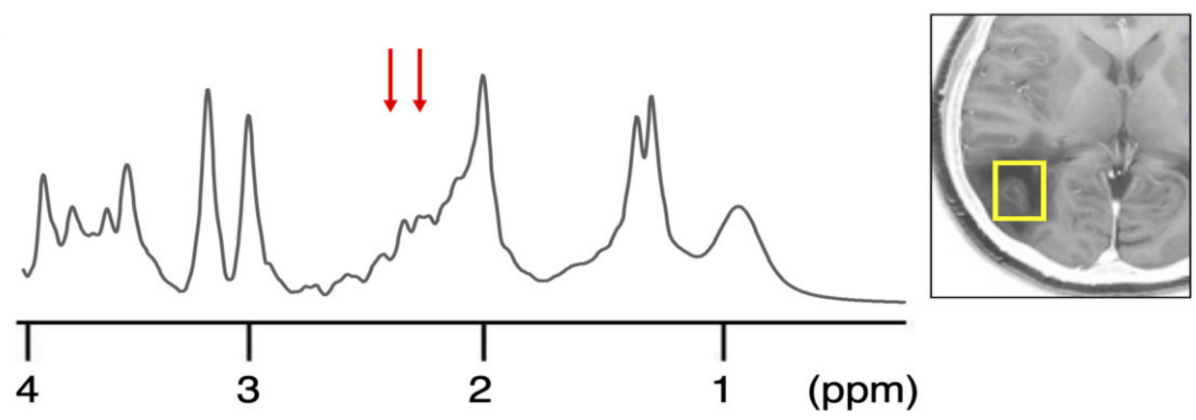

A $T$ T G G C A G G C A C G C C

B
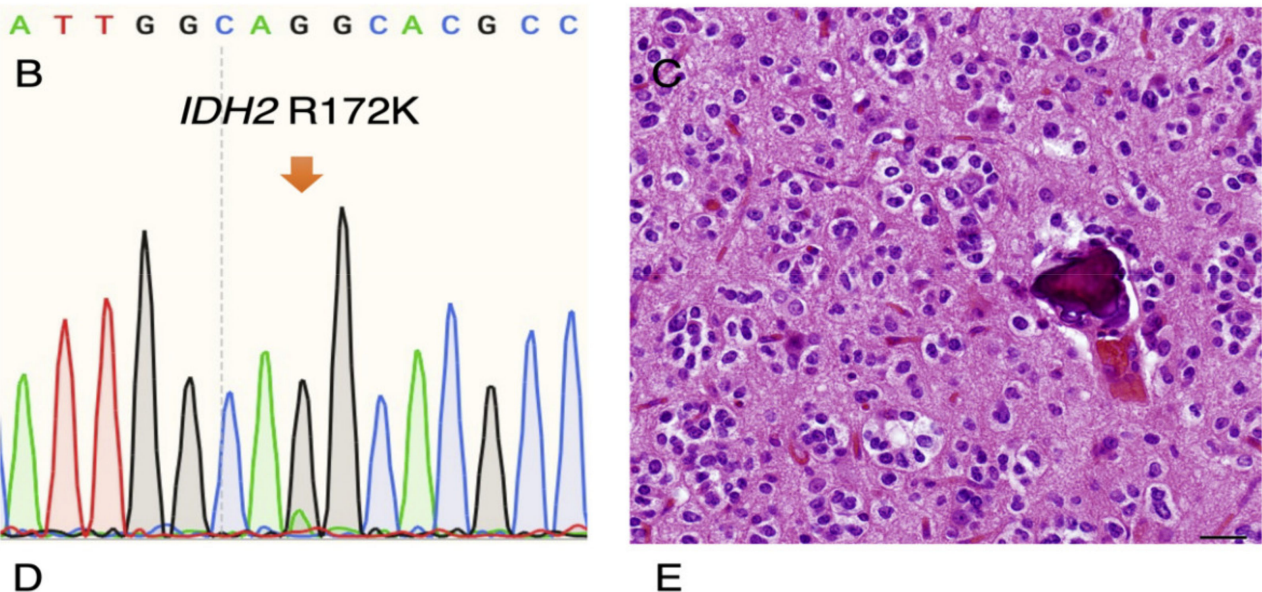

E
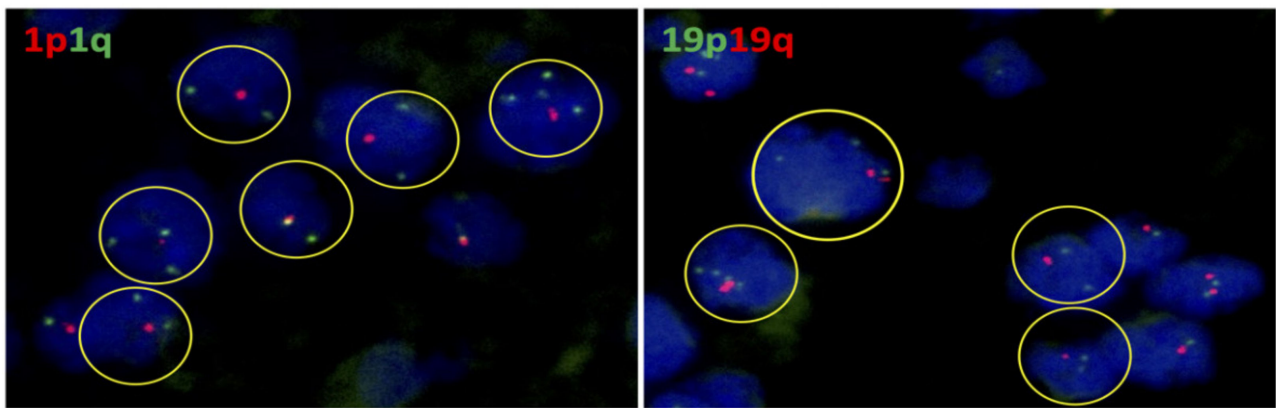

Figure 2. IDH2 mutation identified in a second case. (A) SVMRS spectra show minimal peaks at $2.25 \mathrm{ppm}$ (red arrows) and 2HG quantified to be $2.763 \mathrm{mM}$. (B) A small, IDH2 R172K-mutant peak was detected. (C) Morphologically, tumor cells with perinuclear halos lacking astrocytic processes, chicken-wire vessels and calcification were observed, suggestive of oligodendroglioma (scale bar $=20 \mu \mathrm{m}$ ). Codeletion of $1 \mathrm{p}(\mathrm{D})$ and 19q (E) were detected by fluorescence in situ hybridization (FISH). 
Thus, we found that the MRS was correct, and that we initially failed to detect rare IDH2 mutations in two out of the five cases. The specificity of 2HG, at a cutoff of $1.489 \mathrm{mM}$ to determine IDH mutation, was calculated to be $81.3 \%$, not $72.2 \%$, as originally reported [1]. The demographics of the five cases are summarized below (Table 1).

Table 1. Characteristics of the 5 presumed, false-positive cases.

\begin{tabular}{cccccc}
\hline Case No. & $\mathbf{1}$ & $\mathbf{2}$ & $\mathbf{3}$ & $\mathbf{4}$ & $\mathbf{5}$ \\
\hline Age & 30 & 51 & 59 & 72 & 67 \\
Sex & $\mathrm{F}$ & $\mathrm{F}$ & $\mathrm{M}$ & $\mathrm{F}$ & $\mathrm{F}$ \\
Location & Rt Frontal & Rt Parietal & Rt Frontal & Rt Temporal & Lt Thalamus \\
2HG $(\mathrm{mM})$ & 6.820 & 2.763 & 5.589 & 4.477 & 5.448 \\
Lactate $(\mathrm{mM})$ & 1.912 & 4.824 & 0.189 & 0.000 & 6.874 \\
IDH1 R132H IHC & Negative & Negative & Negative & Negative & Negative \\
IDH 1/2 sequence & IDH2 R172W & IDH2 R172K & WT $^{6}$ & WT & N/A \\
Mutant peak & Small & Small & None & None $^{4}$ & N/A \\
1p/19q FISH & 1p/19q codel & 1p/19q codel & N/A & N/A $^{4}$ & N/A $^{4}$ \\
Pathological & OD $^{5}$ & OD $^{5}$ & DA $^{2}$ & GBM $^{3}$ & DA $^{2}$ \\
diagnosis & & & & \\
\hline
\end{tabular}

${ }^{1}$ codeleted $;{ }^{2}$ diffuse astrocytoma; ${ }^{3}$ glioblastoma; ${ }^{4}$ not available/not applicable; ${ }^{5}$ oligodendroglioma; ${ }^{6}$ wildtype.

We compared the metabolite concentrations of true-positive cases $(n=2)$ and falsepositive cases $(n=3)$ (Table 2). Due to the small number of cases, the median concentration of metabolites between the two groups did not reach statistical significance. However interestingly, we found that the median GSH and Glu+Gln concentrations were lower, and the median Lac concentration was higher in the true-positive group than in the falsepositive group, which is consistent with data that we $[1,6]$ and others $[7,8]$ have previously reported in IDH-mutant versus IDH-wildtype gliomas.

Table 2. Comparison of metabolites between true-positive and false-positive cases.

\begin{tabular}{|c|c|c|c|}
\hline Metabolite & $\begin{array}{l}\text { Median Concentration }(\mathrm{mM}) \\
\text { True-Positive Cases }(n=2)\end{array}$ & $\begin{array}{l}\text { Median Concentration }(\mathrm{mM}) \\
\text { False Positive Cases }(n=3)\end{array}$ & $p$-Value \\
\hline $\mathrm{GSH}^{1}$ & 1.020 & 1.839 & 0.40 \\
\hline $2 \mathrm{HG}^{2}$ & 4.792 & 5.448 & $>0.99$ \\
\hline $\mathrm{mI}^{3}$ & 5.561 & 3.862 & 0.40 \\
\hline $\mathrm{Lac}^{4}$ & 4.393 & 0.189 & 0.40 \\
\hline $\mathrm{tCh}^{5}$ & 1.563 & 1.860 & 0.80 \\
\hline tNAA $^{6}$ & 3.165 & 4.085 & 0.40 \\
\hline $\mathrm{tCr}{ }^{7}$ & 3.803 & 5.890 & 0.80 \\
\hline $\mathrm{Glu}+\mathrm{Gln}^{8}$ & 5.055 & 12.35 & 0.20 \\
\hline
\end{tabular}

\section{Discussion}

In the present study, we re-evaluated five gliomas initially assessed to be of IDH wildtype but showed a high accumulation of $2 \mathrm{HG}$ and were thought to be false-positive. Two cases harbored rare IDH2 R172 mutations that were not detected in the original analysis. The 2HG molecule contains five nonexchangeable protons, giving rise to multiplets at three locations on MRS: of approximately 4.02, 2.25, and $1.90 \mathrm{ppm}$ [9]. The largest multiplet is located at $2.25 \mathrm{ppm}$. The detection of this multiplet is complicated by the spectral overlap of glutamate (Glu; $2.43 \mathrm{ppm}$ ), glutamine (Gln; $2.34 \mathrm{ppm}$ ), and gamma-aminobutyric acid (GABA; $2.28 \mathrm{ppm}$ ), all of which share the ${ }^{4} \mathrm{CH}_{2}$ group [10]. This can be expected given the structural similarities of Glu, Gln and 2HG. The direct detection of the multiplet at $1.90 \mathrm{ppm}$ is made difficult due to its proximity to the NAA resonance at $2.01 \mathrm{ppm}$, which shares ${ }^{3} \mathrm{CH}_{2}$. Finally, the multiplet at 4.02 partially overlaps with creatine (Cr: $3.92 \mathrm{ppm}$ ), phosphocreatine (PCr; $3.94 \mathrm{ppm}$ ), myoinositol (Ins; $4.06 \mathrm{ppm}$ ), lactate (Lac; $4.1 \mathrm{ppm}$ ) and free 
choline (fCh; $4.05 \mathrm{ppm}$ ), sharing ${ }^{2} \mathrm{CH}_{2}$ [9], which makes the unambiguous detection of $2 \mathrm{HG}$ challenging. A false-positive rate of approximately $22 \%$ was observed by Pope et al. using short-echo MRS (TE at $30 \mathrm{~ms}$ ) for the detection of 2HG in gliomas [11]. This false-positive rate can be reduced by using long-echo MRS with TE at $97 \mathrm{~ms}$ and three-dimensional volume-localized basis (VLB) spectra, minimizing the effect of macromolecules in the detection of $2 \mathrm{HG}[9,12]$. Other studies suggest that $2 \mathrm{HG} /[\mathrm{lipid}+$ lactate] ratios [8] or a combination of $2 \mathrm{HG}$ and Glu [7] can provide a higher diagnostic accuracy than $2 \mathrm{HG}$ alone.

The sampling error must be considered when assessing the DNA of tumor tissues, especially when using frozen tissue. A recent study by Barritault et al. detected IDH1 and TERT promoter mutations from non-diagnostic biopsies from glioma patients using SNaPshot polymerase chain reaction [13], suggesting that methods that are more sensitive than Sanger sequencing may detect mutations in tissues with a low tumor volume. Since the mutant peak was small in both cases with detected IDH2 mutation, we suspect that the tumor volume was low in these cases.

The limitations of this study include the small number of subjects, a suboptimal analysis of $2 \mathrm{HG}$ at a TE of $30 \mathrm{~ms}$, the lack of ample tissue for DNA testing in one case, and an inability to detect rare IDH1/2 mutations differentiated from IDH1 R132 and IDH2 R172, such as IDH1 R100Q. Thus, we were unable to determine the exact cause of the false-positive results in the remaining three cases.

One of the advantages of detecting $2 \mathrm{HG}$ by MRS is the non-invasive screening for rare mutations of IDH1 and IDH2, as all IDH mutations are known to produce 2HG [14]. Highly reliable antibodies for IDH1 R132H, which constitutes approximately $90 \%$ of all IDH mutations in gliomas [3,15], are commercially available and widely used [16,17], but antibodies for other IDH1 and IDH2 mutant proteins are less widely available [18]. A recent, multicenter study suggested that non-IDH1-R132H IDH1/2 mutations are associated with an improved survival for astrocytomas compared to their R132H-mutant counterpart [19], which provides a basis for analyzing these rare mutations. In the present study, rare IDH2 mutations were found in 2 out of 5 cases initially thought to be false-positive for $2 \mathrm{HG}$. Both cases were pathologically diagnosed as oligodendrogliomas, which are known to almost uniformly harbor IDH mutations and $1 \mathrm{p} / 19 \mathrm{q}$ codeletions [5].

Author Contributions: Conceptualization, M.N., H.I.; methodology, M.N., H.I., K.M., K.O.; formal analysis, M.N., H.I., K.M.; investigation, M.N., R.G., H.T., R.O., J.W., Y.T., K.O.; writing-original draft preparation, M.N.; writing—review and editing, H.I., R.G., K.O., A.K.; supervision, H.I., T.N., Y.F. All authors have read and agreed to the published version of the manuscript.

Funding: This research received no external funding.

Institutional Review Board Statement: Ethical approval was obtained in accordance with the human research guidelines of the Institutional Review Board of Niigata University (Approval \#2017-0163, approval date is 11 September 2017).

Informed Consent Statement: Written informed consent was obtained from all participants involved in the study.

Data Availability Statement: The datasets analyzed during the current study are available from the corresponding author upon request.

Acknowledgments: We would like to acknowledge all who helped with imaging, Ken Ohno for assistance in imaging analysis, Sumihito Nobusawa of Department of Human Pathology, Gunma University Graduate School of Medicine, for providing information about primers and Shingo Nigorikawa for technical assistance with molecular analyses.

Conflicts of Interest: The authors declare no conflict of interest. 


\section{References}

1. Natsumeda, M.; Igarashi, H.; Nomura, T.; Ogura, R.; Tsukamoto, Y.; Kobayashi, T.; Aoki, H.; Okamoto, K.; Kakita, A.; Takahashi, H.; et al. Accumulation of 2-hydroxyglutarate in gliomas correlates with survival: A study by 3.0-tesla magnetic resonance spectroscopy. Acta Neuropathol. Commun. 2014, 2, 158. [CrossRef] [PubMed]

2. Provencher, S. Estimation of metabolite concentrations from localized in vivo proton NMR spectra. Magn. Reson. Med. 1993, 30, 672-679. [CrossRef] [PubMed]

3. Hartmann, C.; Meyer, J.; Balss, J.; Capper, D.; Mueller, W.; Christians, A.; Felsberg, J.; Wolter, M.; Mawrin, C.; Wick, W.; et al. Type and frequency of IDH1 and IDH2 mutations are related to astrocytic and oligodendroglial differentiation and age: A study of 1,010 diffuse gliomas. Acta Neuropathol. 2009, 118, 469-474. [CrossRef] [PubMed]

4. Ambros, P.F.; Ambros, I.M. Pathology and biology guidelines for resectable and unresectable neuroblastic tumors and bone marrow examination guidelines. Med. Pediatr. Oncol. 2001, 37, 492-504. [CrossRef] [PubMed]

5. Louis, D.N.; Ohgaki, H.; Wiestler, O.D.; Cavenee, W.K.; Ellison, D.W.; Figarella-Branger, D.; Perry, A.; Reifenberger, G.; von Deimling, A. WHO Classification of Tumours of the Central Nervous System; IARC: Lyon, France, 2016.

6. Natsumeda, M.; Motohashi, K.; Igarashi, H.; Nozawa, T.; Abe, H.; Tsukamoto, Y.; Ogura, R.; Okada, M.; Kobayashi, T.; Aoki, H.; et al. Reliable diagnosis of IDH-mutant glioblastoma by 2-hydroxyglutarate detection: A study by 3-T magnetic resonance spectroscopy. Neurosurg. Rev. 2018, 41, 641-647. [CrossRef] [PubMed]

7. Nagashima, H.; Tanaka, K.; Sasayama, T.; Irino, Y.; Sato, N.; Takeuchi, Y.; Kyotani, K.; Mukasa, A.; Mizukawa, K.; Sakata, J.; et al. Diagnostic value of glutamate with 2-hydroxyglutarate in magnetic resonance spectroscopy for IDH1 mutant glioma. NeuroOncology 2016, 18, 1559-1568. [CrossRef] [PubMed]

8. Suh, C.H.; Kim, H.S.; Park, J.E.; Jung, S.C.; Choi, C.G.; Woo, D.C.; Lee, H.B.; Kim, S.J. Comparative Value of 2-Hydroxyglutarateto-Lipid and Lactate Ratio versus 2-Hydroxyglutarate Concentration on MR Spectroscopic Images for Predicting Isocitrate Dehydrogenase Mutation Status in Gliomas. Radiol. Imaging Cancer 2020, 2, e190083. [CrossRef] [PubMed]

9. Choi, C.; Ganji, S.; Hulsey, K.; Madan, A.; Kovacs, Z.; Dimitrov, I.; Zhang, S.; Pichumani, K.; Mendelsohn, D.; Mickey, B.; et al. A comparative study of short- and long-TE (1)H MRS at $3 \mathrm{~T}$ for in vivo detection of 2-hydroxyglutarate in brain tumors. NMR Biomed. 2013, 26, 1242-1250. [CrossRef] [PubMed]

10. Govindaraju, V.Y.K.; Maudsley, A.A. Proton NMR chemical shifts and coupling constants for brain metabolites. NMR Biomed. 2000, 13, 129-153. [CrossRef]

11. Pope, W.B.; Prins, R.M.; Albert Thomas, M.; Nagarajan, R.; Yen, K.E.; Bittinger, M.A.; Salamon, N.; Chou, A.P.; Yong, W.H.; Soto, H.; et al. Non-invasive detection of 2-hydroxyglutarate and other metabolites in IDH1 mutant glioma patients using magnetic resonance spectroscopy. J. Neurooncol. 2012, 107, 197-205. [CrossRef] [PubMed]

12. Choi, C.; Ganji, S.K.; DeBerardinis, R.J.; Hatanpaa, K.J.; Rakheja, D.; Kovacs, Z.; Yang, X.L.; Mashimo, T.; Raisanen, J.M.; Marin-Valencia, I.; et al. 2-hydroxyglutarate detection by magnetic resonance spectroscopy in IDH-mutated patients with gliomas. Nat. Med. 2012, 18, 624-629. [CrossRef] [PubMed]

13. Barritault, M.; Picart, T.; Poncet, D.; Fenouil, T.; d’Hombres, A.; Gabut, M.; Guyotat, J.; Jouanneau, E.; Ameli, R.; Joubert, B.; et al. Avoiding New Biopsies by Identification of IDH1 and TERT Promoter Mutation in Nondiagnostic Biopsies from Glioma Patients. Neurosurgery 2020, 87, E513-E519. [CrossRef] [PubMed]

14. Gross, S.; Cairns, R.A.; Minden, M.D.; Driggers, E.M.; Bittinger, M.A.; Jang, H.G.; Sasaki, M.; Jin, S.; Schenkein, D.P.; Su, S.M.; et al. Cancer-associated metabolite 2-hydroxyglutarate accumulates in acute myelogenous leukemia with isocitrate dehydrogenase 1 and 2 mutations. J. Exp. Med. 2010, 207, 339-344. [CrossRef] [PubMed]

15. Yan, H.; Parsons, D.W.; Jin, G.; McLendon, R.; Rasheed, B.A.; Yuan, W.; Kos, I.; Batinic-Haberle, I.; Jones, S.; Riggins, G.J.; et al. IDH 1 and IDH 2 mutations in gliomas. N. Engl. J. Med. 2009, 360, 765-773. [CrossRef] [PubMed]

16. Capper, D.; Weissert, S.; Balss, J.; Habel, A.; Meyer, J.; Jager, D.; Ackermann, U.; Tessmer, C.; Korshunov, A.; Zentgraf, H.; et al. Characterization of R132H mutation-specific IDH1 antibody binding in brain tumors. Brain Pathol. 2010, 20, 245-254. [CrossRef] [PubMed]

17. Kato, Y.; Jin, G.; Kuan, C.T.; McLendon, R.E.; Yan, H.; Bigner, D.D. A monoclonal antibody IMab-1 specifically recognizes IDH1R132H, the most common glioma-derived mutation. Biochem. Biophys. Res. Commun. 2009, 390, 547-551. [CrossRef] [PubMed]

18. Kato, Y. Specific monoclonal antibodies against IDH1/2 mutations as diagnostic tools for gliomas. Brain Tumor Pathol. 2015, 32, 3-11. [CrossRef] [PubMed]

19. Tesileanu, C.M.S.; Vallentgoed, W.R.; Sanson, M.; Taal, W.; Clement, P.M.; Wick, W.; Brandes, A.A.; Baurain, J.F.; Chinot, O.L.; Wheeler, H.; et al. Non-IDH1-R132H IDH1/2 mutations are associated with increased DNA methylation and improved survival in astrocytomas, compared to IDH1-R132H mutations. Acta Neuropathol. 2021, 141, 945-957. [CrossRef] [PubMed] 